\title{
A three-dimensional numerical simulation of particle-gas mixing in an ejection with a spherical sample surface
}

\author{
K. Liu, L. Li, S. Yuan, J. Bai \& P. Li \\ Institute of Fluid Physics, China Academy of Engineering Physics, \\ Mianyang, China
}

\begin{abstract}
A three-dimensional numerical simulation employed with particle trajectory model is implemented to simulate particle-gas interaction in spherical ejecta problem, in which particles are ejected from a shocked sample surface. The particles' initial distribution is modelled due to characteristics of ejection processes and references data. And action on gas of the moving sample surface is simulated by using ghost fluid coupling Eulerian-Lagrangian method. Only a steady drag force between particles and gas is considered at present. The evolutions of gas flow and particle movements are shown in this paper. It is concluded that the particle cloud naturally changes into a high-density lowvelocity region and a low-density high-velocity one. This qualitatively agrees with ejection experimental acquaintance.

Keywords: spherical ejecta, particle-gas mixing, particle trajectory model, ghost fluid coupling Eulerian-Lagrangian method, three-dimensional numerical simulation.
\end{abstract}

\section{Introduction}

Ejection is an important issue in shock dynamics [1-4], which happens as strong shock reflects at free surface of metal material sample. Interaction between shock and the inhomogeneous or disfiguration around the free surface will cause the material to form microjets, fragmentations, micro-spallation, shock-melting, unload-melting and other processes, and furtherly form ejection [5]. In ejection, the particles have high velocity (about $1-7 \mathrm{~km} / \mathrm{s}$ ), micro size scale (several micrometers), numerous number, negligible volume to gas flow, and such 
features are largely different from that of the general gas-particle two phase flow occurs in industrial production [6,7]. In addition, shock appears in gas field inevitably during sample moving. The particles mixing with gas in ejection is a typical multi-scale, complex flow problem. However, few researches about this issue have been performed so far. This paper is devoted to obtain the evolution of particle-gas mixing for a specific computational model through numerical simulation, and subsequently to anticipate gaining deeper understanding in principle.

Taking no account of mass and heat exchanging between particles and gas, the numerical simulation of the mixing in ejection can be simply analyzed by utilizing gas-particle two phase flow method [8]. In which, the gas is described by inviscid compressible Eulerian equations and each particle is tracked by Newton's second law. The phases are related through interaction forces. The action on gas from the moving sample surface is modeled by ghost fluid coupling Eulerian-Lagrangian method [9]. For each individual particle, the forces it receives include gravity, gradient pressure force, steady drag, added mass force, Basset force, etc. For simplicity purposes, only drag force is considered. And the correctness factor of compressibility is introduced to original drag coefficient to reflect high Mach number characteristics. The initial location, velocity and size of each ejecta particle are modeled and simplified according to references data $[2,5]$. As gas computation is concerned, third order piecewise parabolic method [11] is adopted. And the body force on each control volume equals to the negative summation of the received forces of all particles located in the volume.

Modeling and numerical method are described in section 2. Computational results are discussed in section 3 . Section 4 is conclusions.

\section{Modelling and numerical methods}

\subsection{Modelling}

In numerical simulation of gas-particle mixing, we assume particles are spherical and collisionless. And formation time of particles is neglected, which means that all particles are ejected in sufficient small interval as shock reflects at free surface and this interval are far less than the characteristic time of particle movement. The ejecta surface or sample surface is spherical and with initial radius of $4.2 \mathrm{~cm}$. The velocity and radius variation with respect to time is shown in figure 1 , which is obtained by executing a one-dimensional Lagrangian explosion and shock hydrocode. Initially, the spherical space surrounded by sample is filled with diatomic gas with 1 bar pressure and $10^{-3} \mathrm{~g} / \mathrm{cm}^{3}$ density. All particles are reflected stiffly at each boundary except sample surface. 


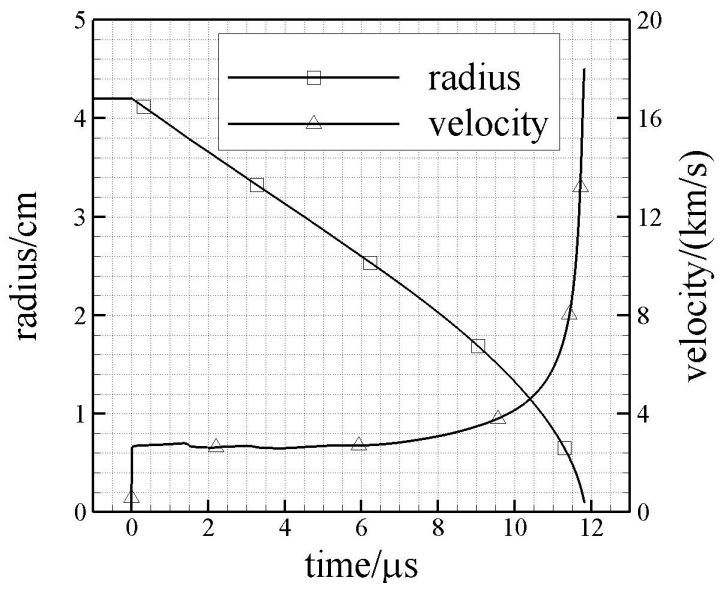

Figure 1: $\quad$ The variation of sample surface velocity and radius versus time.

We utilize the experimental data as shown in figure 2b) in reference [5] to determine the total mass and velocity distribution of ejecta particles. Assuming in time interval of $t$ to $t+\Delta t$, the total mass of ejecta material changes from $M$ to $M+\Delta M$, then the velocity $u_{\mathrm{ej}}$ of all particles belong to this $\Delta M$ part equals to $L_{\mathrm{f}} / t$, where $L_{\mathrm{f}}$ is the initial distance between sample surface and Asay foil which is $2.74 \mathrm{~mm}$ as experiment sets.

On the other hand, by making use of the experimental results of reference [2] we can obtain the particle size distribution. The experimental results show that the particles number $N$ and the particles diameter $D$ satisfies a power law relationship of $N(D)=N_{0} D^{-\kappa}$, where $N_{0}$ is a constant to be determined and $\kappa$ ranges from 4.5 to 6 according to three-dimensional percolation theory. We chose $\kappa=5.5$ for the case of fully melted sample surface. In addition, from the mass conservation there is relation

$$
\Delta M=\frac{1}{6} \rho_{\mathrm{p}} \pi \int_{0}^{\infty} N_{0} D^{3-\kappa} \mathrm{d} D,
$$

where $\rho_{\mathrm{p}}$ is the density of particle material and equals to $2.7 \mathrm{~g} / \mathrm{cm}^{3}$ for aluminum. From equation (1) the total particle number corresponds to the ejecta material with initial velocity $u_{\mathrm{ej}}$ and mass $\Delta M$ can be determined. For simplicity, to reduce the particles that join the practical calculation, the smallest and largest sizes of particles are $2 \mu \mathrm{m}$ and $30 \mu \mathrm{m}$, respectively. The particles are uniquely distributed on the sample surface at beginning time of calculation with random algorithm. In spherical coordinate system, the coordinates of an arbitrary particle can be written as

$$
(r, \theta, \varphi)=\left(R_{\mathrm{I}}, 2 \arccos \varepsilon, 2 \pi \varepsilon\right),
$$

where $r, \theta, \varphi$ are the radius, polar angle and azimulthal angle of particle center, respectively, $R_{\mathrm{I}}$ is the initial radius of sample surface, $\varepsilon$ is a random value between $[0,1]$. 
Figure 2 illustrates an example of the initial spatial distribution of particles from the above method. For visible purpose, the particle size are absolutely amplified by 75 times in figure 2a) and 25 times in figure 2b), respectively. The red lines in figure $2 b$ ) are the meshes on the sample surface.
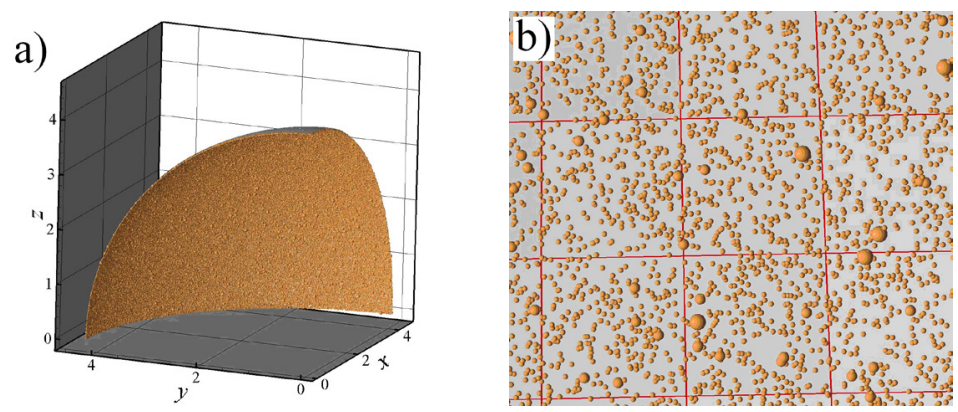

Figure 2: $\quad$ The illustration of initial sample location and particles distribution.

One more thing to be illuminated is that the total particles number is about $1.05 \times 10^{11}$ according to the references data and relation (1). This number is too large to be considered feasibly at present. So, in practical computation, the mass of ejecta material is about $3.6 \times 10^{-6}$ times to experiments data, the corresponding particles number is about $3.8 \times 10^{5}$.

\subsection{Gas-particle two phase flow method}

A particle trajectory model of gas-particle two phase flow is implemented to simulate the interaction and evolution of ejecta particles and gas flow. The particle volume is neglected in calculation. In spherical coordinate system, Lagrangian equations governing the heat free inviscid gas flow is as follows

$$
\begin{gathered}
\frac{\mathrm{d} \rho}{\mathrm{d} t}+\frac{\rho}{r^{2}} \frac{\partial\left(r^{2} u\right)}{\partial r}+\frac{\rho}{r \sin \theta} \frac{\partial(\sin \theta v)}{\partial \theta}+\frac{\rho}{r \sin \theta} \frac{\partial w}{\partial \varphi}=0 \\
\frac{\mathrm{d} u}{\mathrm{~d} t}=f_{r}-\frac{1}{\rho} \frac{\partial p}{\partial r} \\
\frac{\mathrm{d} v}{\mathrm{~d} t}=f_{\theta}-\frac{1}{\rho r} \frac{\partial p}{\partial \theta} \\
\frac{\mathrm{d} w}{\mathrm{~d} t}=f_{\varphi}-\frac{1}{\rho r \sin \theta} \frac{\partial p}{\partial \varphi} \\
\frac{\mathrm{d} e}{\mathrm{~d} t}=-\frac{p}{\rho}\left(\frac{1}{r} \frac{\partial(r u)}{\partial r}+\frac{1}{r} \frac{\partial v}{\partial \theta}+\frac{1}{r \sin \theta} \frac{\partial w}{\partial \varphi}\right)
\end{gathered}
$$


where $u, v, w, f_{\mathrm{r}}, f_{\theta}, f_{\varphi}$ are the velocity and body force components in $r, \theta$, $\varphi$ directions, respectively, and $e$ the specific initial energy. For ideal gas, the equation of state is

$$
p=(\gamma-1) \rho e
$$

System of (3)-(7) can be converted to three one-dimensional problems with dimension splitting method. To solve each one-dimensional problem, a Lagrangian-remapping type piecewise parabolic method (PPM) is applied. PPM can be divided into four steps: 1) the piecewise parabolic interpolation of physical quantities; 2) solving Riemann problems approximately at cells interface; 3) marching of Lagrange equations; and 4) remapping the physical quantities onto stationary Euler meshes. The algorithm detail refers to [10].

We use Newton's second law to track each particle. That is

$$
m_{\mathrm{p}} \frac{\mathrm{d} \mathbf{u}_{\mathrm{p}}}{\mathrm{d} t}=\mathbf{F}_{\text {total }}
$$

in which $m_{\mathrm{p}}$ is the particle mass, $\mathbf{u}_{\mathrm{p}}$ the particle velocity vector, $\mathbf{F}_{\text {total }}$ the all forces that the particle suffers, including gravity, Lorentz force, drag force, added mass force, Basset force, buoyancy force and etc. In this paper, only drag force is under consideration. To conveniently update particle status, the action force on a particle is decomposed in Cartesian coordinate system. Therefore, the body force of gas in a control volume shown in equations (3)-(7) and the drag force of a particle have the relation

$$
\mathbf{f}=-\mathbf{A} \frac{\sum_{n=1}^{N} \mathbf{F}_{\mathrm{n}}^{\mathrm{D}}}{\rho \Delta V}
$$

In equation (10), the minus sign denotes force interplay relation, $N$ is the particles number the cell contains, $\rho$ is gas density, $\Delta V$ is the cell volume, $\mathbf{A}$ is the adjoint matrix from Cartesian system to spherical system which can be written as

$$
\mathbf{A}=\left(\begin{array}{ccc}
\sin \theta \cos \varphi & \sin \theta \sin \varphi & \cos \theta \\
\cos \theta \cos \varphi & \cos \theta \sin \varphi & -\sin \theta \\
-\sin \varphi & \cos \varphi & 0
\end{array}\right)
$$

For a single particle, the drag force is

$$
\mathbf{F}^{\mathrm{D}}=\frac{1}{2} C_{\mathrm{D}} \rho\left|\mathbf{u}-\mathbf{u}_{\mathrm{p}}\right|\left(\mathbf{u}-\mathbf{u}_{\mathrm{p}}\right) S
$$

where $C_{\mathrm{D}}$ is the drag coefficient, $S$ is the particle reference area, for spherical particle $S=\pi r_{\mathrm{p}}^{2}, r_{\mathrm{p}}$ is the particle radius. Drag coefficient is related to Reynolds number ( $\operatorname{Re}=2 \rho \mathrm{r}_{\mathrm{p}} \mid \mathbf{u}-\mathbf{u}_{\mathrm{p}} / \mu, \mu$ is dynamic viscous coefficient of gas) and has different formulations in different Reynolds regions 


$$
C_{\mathrm{D}}=\left\{\begin{array}{cc}
24 / \operatorname{Re}(1+3 / 16 \mathrm{Re}) & \operatorname{Re}<0.49 \\
24 / \operatorname{Re}\left(1+0.15 \mathrm{Re}^{0.687}\right) & 0.49 \leq \mathrm{Re}<1300 \\
0.4 & \operatorname{Re} \geq 1300
\end{array}\right.
$$

In practical application, if accounting for strong compressibility of gas, remedy needs to add on standard drag coefficient formula

$$
C_{\mathrm{DC}}=C_{\mathrm{D}} f_{\mathrm{c}}(\mathrm{Mr})
$$

where

$$
f_{c}(\mathrm{Mr})=\frac{1+\frac{\mathrm{Mr}}{\mathrm{Re}}\left[3.82+1.28 \exp \left(-\frac{1.25 \mathrm{Re}}{\mathrm{Mr}}\right)\right]}{1+\exp \left[-\frac{0.427}{\mathrm{Mr}^{4.63}}-\frac{3}{\mathrm{Re}^{0.88}}\right]}
$$

$\mathrm{Mr}=\left|\mathbf{u}-\mathbf{u}_{\mathrm{p}}\right| / a$ is relative Mach number, $a$ is the local sound speed.

\subsection{Ghost fluid coupling Eulerian-Lagrangian algorithm}

In this paper, level set method based ghost fluid coupling Eulerian-Lagrangian algorithm (GFEL) $[9,11]$ is implemented to simulate the interaction between sample surface and gas. GFEL is originally designed to investigate fluid/solid coupling problem by solving Eulerian and Lagrangian equations respectively and making the most of the current hydrocodes. It can give flow field evolution in fluid and formation and shock responsibility or other information in solid at the same time.

The movement principle of the sample surface is previously known by solving a one-dimensional spherical Lagrangian equation. Assuming the displacement and velocity relations are

$$
\begin{gathered}
\mathbf{x}_{\mathrm{fs}}=\mathbf{x}_{\mathrm{fs}}(t) \\
\mathbf{u}_{\mathrm{fs}}=\mathbf{u}_{\mathrm{fs}}\left(\mathbf{x}_{\mathrm{fs}}, t\right)
\end{gathered}
$$

The curve denoted by equation (14) divides the Eulerian flow field into real fluid part and virtual or ghost fluid part. It can be described by a level set function defined by signed distance. Generally, in real fluid side the level set function is set to be negative. The physical quantities in ghost cells are extrapolated from those quantities at sample surface and in the adjacent real fluid cells, which have relations

$$
\left\{\begin{array}{l}
\rho_{\mathrm{g}}=\rho_{\mathrm{e}} \\
\mathbf{u}_{\mathrm{g}}=\left(2 \mathbf{u}_{\mathrm{fs}} \cdot \mathbf{n}-\mathbf{u}_{\mathrm{e}} \cdot \mathbf{n}\right) \mathbf{n}+\left(\mathbf{u}_{\mathrm{e}} \cdot \mathbf{t}\right) \mathbf{t} \\
p_{\mathrm{g}}=p_{\mathrm{e}}
\end{array}\right.
$$

where $\mathbf{n}, \mathbf{t}$ are the unit vectors in gradient and tangential directions of level set function, respectively, the quantities with subscription ' $\mathrm{g}$ ' correspond to those in 
ghost cells, and 'e' to those obtained by marching Eikonal equation. Eikonal equation is

$$
I_{t}+\mathbf{n} \cdot \nabla I=0
$$

where $I$ is the extrapolation variable, which can be pressure, density, velocity or other quantities. To guarantee the physical quantities in ghost fluid cells are extrapolated from the real fluid, we use an upwind-like scheme to solve relation (17). For instance, in $x$ direction, there is

$$
I_{x}= \begin{cases}\frac{I_{i}-I_{i-1}}{x_{i}-x_{i-1}} & n_{x}>0 \\ \frac{I_{i+1}-I_{i}}{x_{i+1}-x_{i}} & n_{x}<0\end{cases}
$$

Equation (17) indicates a pseudo time marching process. The time step and iteration numbers can be artificially controlled.

\section{Results and discussion}

Based on the model and algorithms described in the last section, numerical simulation of particles ejected from spherical sample surface mixing with gas is performed. The computational domain of gas flow field is a 1/8 sphere with $4.5 \mathrm{~cm}$ radius, which is divided into $90 \times 45 \times 45$ structured meshes in three directions. Figure 3 shows three-dimensional images of the sample surface (the gray spherical surface), the particles locations (green spheres) and the gas density distribution in two orthogonal slices. The outer side of sample surface is ghost fluid and will not be discussed here.

As the sample moves towards sphere center, the velocity differences among particles lead the width of mixing zone increasing with respective to time. But the increasing speed falls down because of gas damp effect (Fig. 4). Meanwhile the sample drives gas to move inwards and one convergent spherical shock occurs in gas. From shock relations, the initial shock velocity is about $3.2 \mathrm{~km} / \mathrm{s}$ and is in the range of $2.64-6.85 \mathrm{~km} / \mathrm{s}$ that particles velocities occupy. So one part of particles is in front of the shock and the other part is behind the shock. Equation (11) shows that that for same size and velocity, particles locate in front of the shock suffer larger drag force than those behind the shock. And in the case of same relative velocity, smaller particle has larger acceleration. Therefore, during particles moving inwards, in front of the shock smaller particles will slow down rapidly and be surpassed by the shock. The velocity of these smaller particles is reduced so low that even be pulled up by the sample surface. Only a few large particles with high velocity still keep in front of the shock. Analogous processes also happen in the region behind the shock. Subsequently, the whole particles cloud graduates to a low-density high-velocity region and a highdensity low-velocity region as shown in figure 5. When sample moves inwards, its velocity monotonically increases. Some particles go back to surface and the total number of particle decreases. 


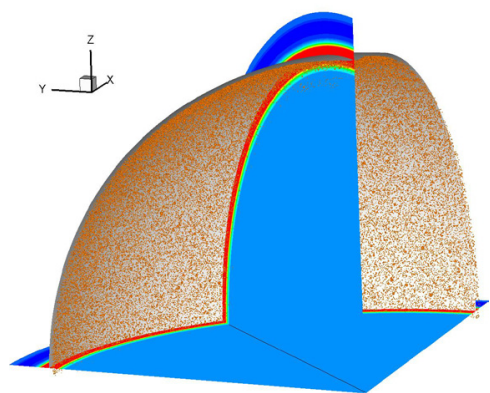

a) $1 \mu \mathrm{s}$

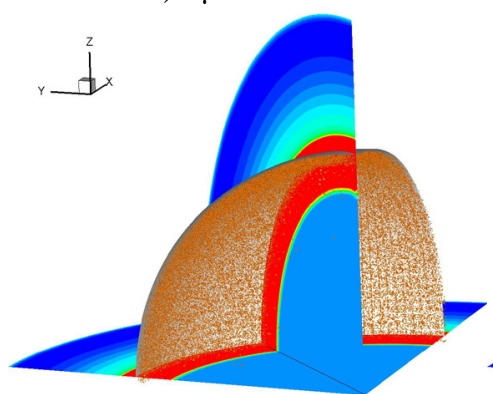

c) $5 \mu \mathrm{s}$

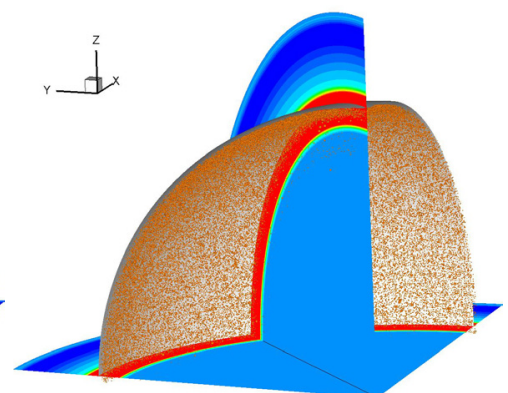

b) $3 \mu \mathrm{s}$

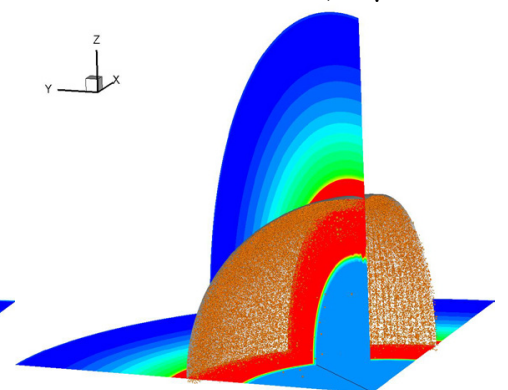

d) $7 \mu \mathrm{s}$

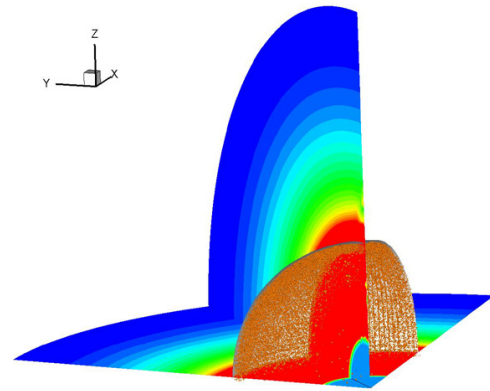

e) $9 \mu \mathrm{s}$

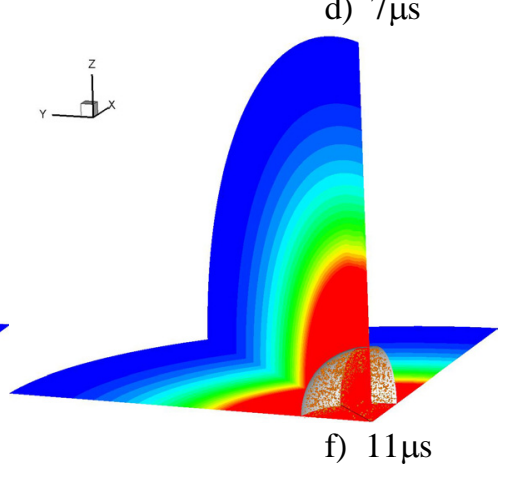

Figure 3: The sample surface and particles locations and gas density distribution at difference moments.

In calculation of the present model, the high-velocity large-size particles that in front of the shock always locate in the front of mixing zone. When those velocities decrease to that of sample surface, the width of mixing zone passes the extramum and begins to decrease. This corresponds to the moments of $8 \mu \mathrm{s}$ in figure 4. As procedure develops with time, some particles go to spherical center and reflect. Particles can be find at all possible radius. And now the width of mixing zone is primarily dominated by the sample surface and decreases with a discontinuous slope. 


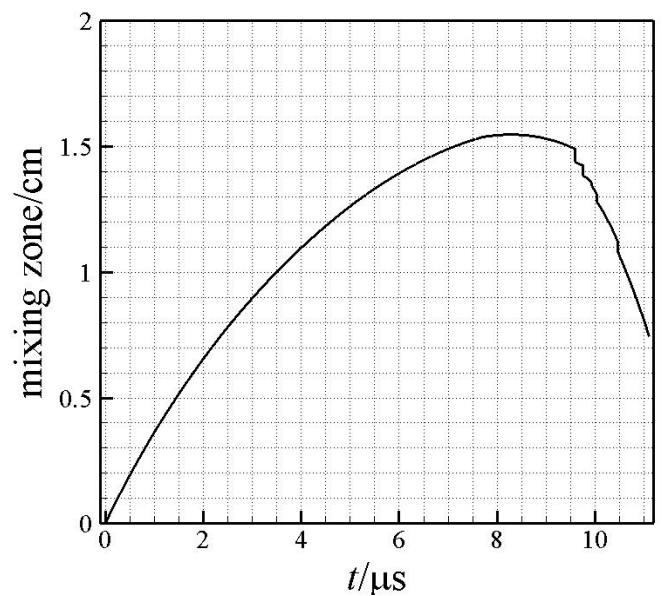

Figure 4: $\quad$ Variation of gas-particle mixing zone versus time.

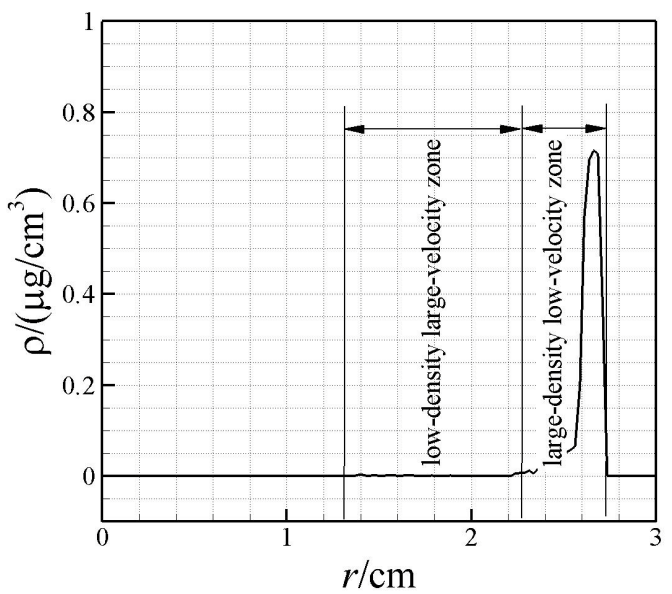

Figure 5: The particle nominal density distribution along radial direction at $11 \mu \mathrm{s}$.

\section{Conclusion}

The ejecta particles initial distribution has been modeled in this paper according to experiments data. A three-dimensional numerical simulation and analysis about evolution of ejecta particles and gas is performed as well. Evolution images of particles and gas flow with moving sample surface are given. The results show that the difference of particles velocities causes the width of mixing zone increasing originally. And under effect of gas damping, particles develop to a low-density high-velocity region and a high-density low-velocity region, which 
is qualitative agree with experimental acquaintance. However the initial particle distribution determined at present is according to the planar ejection experiment data. This is some different from the real case. Furthermore, the effect of ejecta mass, velocity distribution, size distribution, initial gas pressure, sample surface movement, and other respects to evolution of mixing zone need researches in deep.

\section{Reference}

[1] W.S. Vogan, W.W. Anderson, et al. Piezoelectric characterization of ejecta from shocked tin surfaces. Journal of Applied Physics, 2005, 98(113508): $1-10$

[2] D.S. Sorenson, R.W. Minich, et al. Ejecta particle size distributions for shock loaded Sn and Al metals. Journal of Applied Physics, 2002, 92(10): 5830-5835

[3] Jun Chen, Fuqian Jing, et al. Dynamics simulation of ejection of metal under a shock wave. Journal of Physics: Condensed Matter, 2002, 14: 10833-10837

[4] Qifeng Chen, Xiaolin Cao, et al. Parallel molecular dynamics simulations of ejection from the metal $\mathrm{Cu}$ and $\mathrm{Al}$ under shock loading. Chinese Physical Letter, 2005, 22(12): 3151-3154

[5] J.R. Asay, L.P. Mix, F.C. Perry. Ejection of material from shocked surfaces. Applied Physics Letters, 1976, 29(5): 284-287

[6] D.M. Kremer, R.W. Davis, et al. A numerical investigation of the effects of gas-phase particle formation on silicon film deposition from silane. Journal of Crystal Growth, 2003, 247: 333-356

[7] Jianping Zhang, Yong Li, Liang-Shih Fan. Numerical studies of bubble and particle dynamics in a three-phase fluidized bed at elevated pressures. Powder Technology, 2000, 112: 46-56

[8] Kun Liu, Jingsong Bai, Ping Li. Application of particle trajectory model in 1D planar ejection. Journal of Central South University of Technology, 2008, 15(s1): 149-154

[9] M. Arienti, P. Hung, et al. A level set approach to Eulerian-Lagrangian coupling. Journal of Computational Physics, 2003, 185: 213-251

[10] P. Woodward, P. Colella. The numerical simulation of two-dimensional fluid flow with strong shocks, Journal of Computational Physics, 1984, 54: 115-173

[11] T.G. Liu, B.C. Khoo, C.W. Wang. The ghost fluid method for compressible gas-water simulation. Journal of Computational Physics, 2005, 204: 193221 